\title{
Contingencia estructural en el nivel de ingresos: Un análisis en medianas y grandes empresas orenses
}

\section{Structural contingency in the income level: An analysis in medium and large orense companies}

Ingrid Lisseth Batallas Astudillo

Universidad Técnica de Machala, Ecuador

ibatallas1@utmachala.edu.ec

https://orcid.org/0000-0002-8679-9997

Arelys Johanna Luna

Universidad Técnica de Machala, Ecuador

aluna3@utmachala.edu.ec

https://orcid.org/0000-0002-2026-0649

Patricia Alexandra Uriguen Aguirre

Universidad Técnica de Machala, Ecuador

puriguen@utmachala.edu.ec

https://orcid.org/0000-0003-3095-8765

Recepción: 05/02/2021 | Aceptación: 19/04/2021 | Publicación: 10/05/2021

Cómo citar (APA, séptima edición):

Batallas Astudillo, I. L., Luna, A. J. y Uriguen Aguirre, P. A. (2021). Contingencia estructural en el nivel de ingresos: Un análisis en medianas y grandes empresas orenses. Innova Research Journal, 6(2), 209-224. https://doi.org/10.33890/innova.v6.n2.2021.1706

\section{Resumen}

Actualmente, el sector empresarial requiere la implementación sistematizada de estructuras organizativas internas, que permitan a las medianas y grandes empresas expandirse económicamente. La investigación tiene como finalidad analizar el impacto de la gestión del capital humano, estrategias gerenciales y administrativas, y uso eficiente de los recursos como parte de la contingencia estructural de grandes y medianas empresas de la provincia de El Oro. La pesquisa parte de un enfoque cuantitativo de alcance correlacional con diseño transversal, se utilizó el software estadístico Stata 14 para manipular 120 observaciones de la Encuesta Estructural 
Ingrid Batallas Astudillo, Arelys Luna y Patricia Uriguen Aguirre.

ISSN 2477-9024. Innova Research Journal (Mayo-Agosto, 2021). Vol 6, No. 2, pp. 209-224

Empresarial (ENESEM) del Instituto Nacional de Estadística y Censos (INEC) correspondiente a la provincia de El Oro, se midió la variación del nivel de ingresos de las empresas mediante un modelo econométrico de regresión múltiple lineal y pruebas estadísticas de colinealidad, normalidad y homocedasticidad en los residuos. Referente a los resultados, el nivel de ingresos de las organizaciones del sector empresarial depende significativamente del capital humano focalizado en la alta dirección; técnicos administrativos, financieros e informáticos; y del importe y uso eficiente en el consumo de servicios básicos, combustible, suministros, materiales y herramientas. En efecto, la estructura organizativa enfocada en el área de personal calificado y en el correcto manejo de los recursos influye positivamente en el desarrollo económico de medianas y grandes empresas orenses.

Palabras claves: enfoque contingente; nivel de ingresos; optimización de recursos; capital humano; sector empresarial.

\begin{abstract}
Currently, the business sector requires the systematized implementation of internal organizational structures that allow medium and large companies to expand economically. The purpose of this research is to analyze the impact of human capital management, managerial and administrative strategies, and efficient use of resources as part of the structural contingency of large and mediumsized companies in the province of El Oro. The research is based on a quantitative approach of correlational scope with cross-sectional design, the statistical software Stata 14 was used to manipulate 120 observations of the Enterprise Structural Survey (ENESEM) of the National Institute of Statistics and Census (INEC) corresponding to the province of El Oro, the variation of the level of income of the companies was measured through an econometric model of linear multiple regression and statistical tests of collinearity, normality and homoscedasticity in the residuals. Regarding the results, the level of income of the organizations in the business sector depends significantly on the human capital focused on top management; administrative, financial and computer technicians; and the amount and efficient use in the consumption of basic services, fuel, supplies, materials and tools. In fact, the organizational structure focused on the area of qualified personnel and the correct management of resources positively influences the economic development of medium and large companies in the region.

Keywords: contingent approach; income level; resource optimization; human capital; business sector.
\end{abstract}

\title{
Introducción
}

A lo largo de los años, el fenómeno de la globalización ha incidido en la economía mundial, razón por la cual la implementación de nuevas herramientas, procesos y estrategias dentro de los distintos sectores de la economía se hacen necesarias para llevar a cabo un desarrollo sostenible. El sector empresarial es uno de los pilares fundamentales para el crecimiento económico sustentable, las pequeñas, medianas y grandes empresas mediante el control y coordinación de actividades productivas satisfacen los requerimientos de la sociedad, crean valor a las materias primas impulsadas por la innovación y eficiencia en los procesos de producción y aportan a la generación de empleo e ingreso familiar (Delfín y Acosta, 2016). 
Por ello, el análisis de la contingencia estructural como premisa del crecimiento empresarial, según Oña (2020) crea un ambiente organizacional propicio para los trabajadores y empleadores. Sin embargo, la mayoría de las empresas "enfrentan situaciones distintas, contingencias, y requieren diferentes formas de dirección” (Segredo, 2016, p. 591). Por lo tanto, los gerentes como agentes responsables del desempeño empresarial deben implementar estrategias transigentes que permitan al capital humano cumplir las exigencias internas de la organización (Oña, 2020; Segredo, 2016). Para las medianas y grandes empresas es importante realizar actividades de control, tales como, la aplicación de normas y procedimientos, y la implementación de políticas que determinen el cumplimiento de actividades que prevengan riesgos, mismas que deben llevarse a efecto en los diferentes niveles y funciones de la organización (Serrano et al., 2018).

En el caso de Latinoamérica, la principal característica que destaca entre las empresas y organizaciones, es el dinamismo, debido a que las empresas logran resolver de forma eficiente y acertada los enigmas, buscando a la vez modernas oportunidades de negocio, que les permita fortalecerse y adaptarse a los nuevos retos de la realidad empresarial (Hinojosa et al., 2020). En Ecuador, la educación y aprendizaje organizacional se considera un procedimiento clave en la consolidación y desarrollo de las habilidades y destrezas del recurso humano dentro de una empresa, pues la adquisición de nuevos conocimientos es una herramienta indispensable para el continuo mejoramiento organizacional (Luna et al., 2016). El nivel de significancia de la estructura organizacional en el nivel de ingresos de las empresas, se enfoca en el conocimiento de la administración, desarrollo de estrategias, eficiencia y eficacia en la aplicación y control de los recursos de los que dispone la empresa, y en la práctica de mejoras continuas de la estructura organizativa (Ramírez et al., 2017).

El sector empresarial de la provincia de El Oro tiene una actuación regular en su desarrollo, debido a factores como el uso inadecuado de los recursos, falta de personal técnico especializado, acompañado de una endeble organización; por ende, el objetivo de la investigación se centra en analizar la incidencia de la gestión del capital humano, el manejo de estrategias gerenciales y administrativas y el uso óptimo de los recursos como parte de la contingencia estructural en la variación de los ingresos de las medianas y grandes empresas orenses.

Según los resultados de la investigación de Carvallo et al. (2020) únicamente el 19,8\% de las pequeñas y medianas empresas de la provincia de El Oro poseen la documentación de los distintos procedimientos para la administración de sus funciones; hecho que hace necesario realizar una investigación, utilizando un modelo de regresión múltiple lineal y pruebas estadísticas para ahondar en la temática, lo que permitirá determinar de que la contingencia estructural incide en el nivel de ingreso de las medianas y grandes empresas de la provincia de El Oro.

\section{Marco Teórico}

\section{Enfoque contingente}

El enfoque contingente surge como requisito de hacer válidos distintos modelos organizacionales que son considerados mayormente eficientes y flexibles por las empresas, esto debido a que deben adaptarse a los cambios al interactuar con el medio en el que se encuentren 
Ingrid Batallas Astudillo, Arelys Luna y Patricia Uriguen Aguirre.

ISSN 2477-9024. Innova Research Journal (Mayo-Agosto, 2021). Vol 6, No. 2, pp. 209-224

(Méndez et al., 2018). La gestión administrativa y la estructura organizacional están inmiscuidas en la teoría de la contingencia, este enfoque permite obtener información de acuerdo a las condiciones contextuales internas y al desempeño de las funciones para planificar y dirigir la gestión del talento humano y alcanzar la efectividad empresarial (King y Vaiman, 2019).

Valle et al. (2017) mencionan que la diferenciación e integración son consideradas parte del enfoque contingente, siendo un diseño singular y organizacional direccionado a la actuación laboral con la misma participación y coordinación. De hecho, el medio ambiente, estructura organizacional interna, tamaño de empresa, implementación tecnológica y estrategias forman parte del enfoque contingente con la finalidad de lograr una conciliación consistente para alcanzar las metas empresariales (Facin et al., 2016).

Según Graneroa et al. (2017) el enfoque de contingencia mantiene como idea principal la eficiencia y la eficacia de las organizaciones mediante las relaciones organizacionales y los eventos que la subordinan. Dias et al. (2017) expresan que la teoría de contingencia supone que el entorno en el que opera la organización tiene influencia; en consecuencia, se dan cambios en el contexto interno, como la estructura, el tamaño y la estrategia. Uriguen et al. (2018) corroboran que el tamaño de la empresa se determina por los ingresos brutos anuales y la capacidad de plazas de empleo, por ello hoy en día las estructuras organizacionales son flexibles y dinámicas, su estructura organizacional depende de la disposición y usufructo adecuado de los recursos y la interrelación con la gestión del talento humano.

\section{Estructura organizacional}

Las organizaciones tienen como factor elemental una estructura organizacional adecuada y ostensible, en tanto que, se considera una disposición internacional en el desempeño de funciones, donde cada integrante asume un rol que debe cumplir con un máximo rendimiento, para alcanzar las metas previamente fijadas (Bravo et al., 2016). Este pensamiento considera a las empresas no simplemente como edificaciones, ya que el capital humano y las relaciones interpersonales también forman parte de ella (Blanco et al., 2020). Las organizaciones deben llevar a efecto procesos de mejora continua, orientando y procurando el éxito de la empresa, para ello deben proyectar una visión estratégica que permita la reducción de costos y gastos de la empresa (Montoya y Boyero, 2016); para mantener un equilibrio, ordenar y asignar de forma eficiente los recursos con la finalidad de lograr soluciones viables (Duran et al., 2017).

La actuación de los profesionales forma parte del desarrollo y crecimiento de la empresa a través de normas y principios (Hernández, 2016). El cargo de los directores, gerentes, administrativos, financieros y de apoyo logístico dentro de la estructura organizativa empresarial desempeña un rol predominante en la adaptación a los nuevos contextos laborales, la dirección administrativa se sustenta en la aplicación del liderazgo, esto debido a que, las empresas independientemente de su tamaño tienen en común la actuación de un grupo profesionales que se encargan de las tareas de planificación, liderazgo, comunicación y producción (Galarza et al., 2017). Las estrategias gerenciales y administrativas son otros de los factores que forman parte de una estructura organizativa, según Hernández (2016) las estrategias dependen del grado de conocimiento y entendimiento filosófico de la alta dirección de la organización, dado que es la 
encargada de transmitir la cultura y filosofía empresarial a los trabajadores, ambos elementos deben cumplir lineamientos para un apropiado desarrollo de las operaciones. Estas estrategias son un sistema de principios equilibrado entre el contexto interno y externo del sector empresarial que deben ponerse en práctica diariamente (Cedeño et al., 2019).

De acuerdo con Gonzáles (2016) la innovación y el manejo de la tecnología son herramientas que mantienen una tendencia significativa en las empresas, de hecho, permiten desempeñar las actividades productivas eficientemente, de modo que la inserción de profesionales técnicos en equipo informático, y en procesamiento de base de datos dentro de las organizaciones es una actividad eminente. Las empresas para generar productividad requieren del uso eficiente y responsable de los recursos naturales, materiales y tecnológicos, tales como, agua, energía eléctrica, combustibles, lubricantes, suministros, herramientas y repuestos. Vivar et al. (2020) mencionan que es esencial mantener ventaja competitiva, permanecer, expandirse y posicionarse en el mercado mediante la gestión en optimización de los recursos, evitando el despilfarro de materiales, suministros y repuestos, uso inadecuado de las herramientas de trabajo y disponibilidad de tiempo en realizar las actividades. Esta gestión permite satisfacer los requerimientos de los consumidores, generar seguridad en el personal, y generar rentabilidad y beneficios a los empresarios.

\section{Nivel de Ingresos en medianas y grandes empresas}

En gran parte, las personas han creado empresas con la finalidad de generar un máximo en su nivel de ingresos, haciendo posible lograr la rentabilidad a través de un uso eficiente de los recursos (De la Torre, 2018). Lograr la optimización de las actividades operacionales les permitirá a las empresas obtener mayores ganancias. Ponce et al. (2017) señalan que las capacidades y habilidades laborales, y los recursos otorgan dirección elemental y son fuente elemental de rentabilidad para la empresa. Zapata (2015) afirma que la rentabilidad socioeconómica empresarial depende de ajustes organizacionales dentro de un marco coyuntural interno y externo. Por tanto, el tamaño en la estructura empresarial radica en implementar variables de diseño de estructura organizativa; Zapata et al. (2016) mencionan que dichas variables son: centralización, formalización y especialización. Palacio y Núñez (2020) complementan que el tamaño de la empresa se relaciona estrechamente con el importe de los ingresos anuales y la cantidad de trabajadores que posee la misma. Esto es debido a las numerosas actividades y competitividad en distintos elementos y departamentos estratégicos (Ramírez et al., 2018).

Galarza et al. (2017) mencionan que, en países de América Latina, incluido Ecuador las pequeñas y medianas empresas que buscan posicionarse en el mercado nacional e internacional deben enfrentarse a desafíos que dificultan por completo su desempeño empresarial, dado a la fluctuante coyuntura política y económica, y al alto grado de demanda de capital humano calificado. Sotomayor et al. (2018) mencionan que la participación productiva de la provincia de El Oro aporta al crecimiento económico nacional, dado a la abundancia de los recursos naturales, sin embargo, se limita al desarrollo manufacturero, debido a que las empresas se enfocan en incrementar las actividades del sector primario.

De acuerdo con Código Orgánico de la Producción, Comercio e Inversión (2018) las medianas empresas en Ecuador son aquellas que poseen 50 a 199 trabajadores y oscilan ventas 
Ingrid Batallas Astudillo, Arelys Luna y Patricia Uriguen Aguirre.

ISSN 2477-9024. Innova Research Journal (Mayo-Agosto, 2021). Vol 6, No. 2, pp. 209-224

anuales entre $\$ 1 ' 000.001,00$ y $\$ 5^{\prime} 000.000,00$ de dólares americanos mientras que las grandes empresas constan con más de 200 trabajadores y estiman ingresos brutos anuales superiores a los $\$ 5^{\prime} 000.001,00$. En el 2018, las provincias de Pichincha, Guayas, Azuay, Manabí y El Oro se posicionaron en el mercado con un porcentaje del $88.71 \%$, las grandes empresas ecuatorianas crearon 1'189.741 empleos logrando el 71.97\% de ventas, en cambio las medianas empresas A y medianas empresas B generaron correspondientemente 285.517 y 236.038 plazas de trabajo unificando el $15.80 \%$ en ventas (INEC, 2019). Según datos del INEC (2019) determina que las medianas y grandes empresas son el núcleo de inserción laboral y predominan en las ventas anuales, ciertamente la grande empresa expende significativamente el $72.31 \%$, y las medianas empresas A y B representaron ventas del 15.83\%.

\section{Figura 1}

Proporción de medianas y grandes empresas en Ecuador

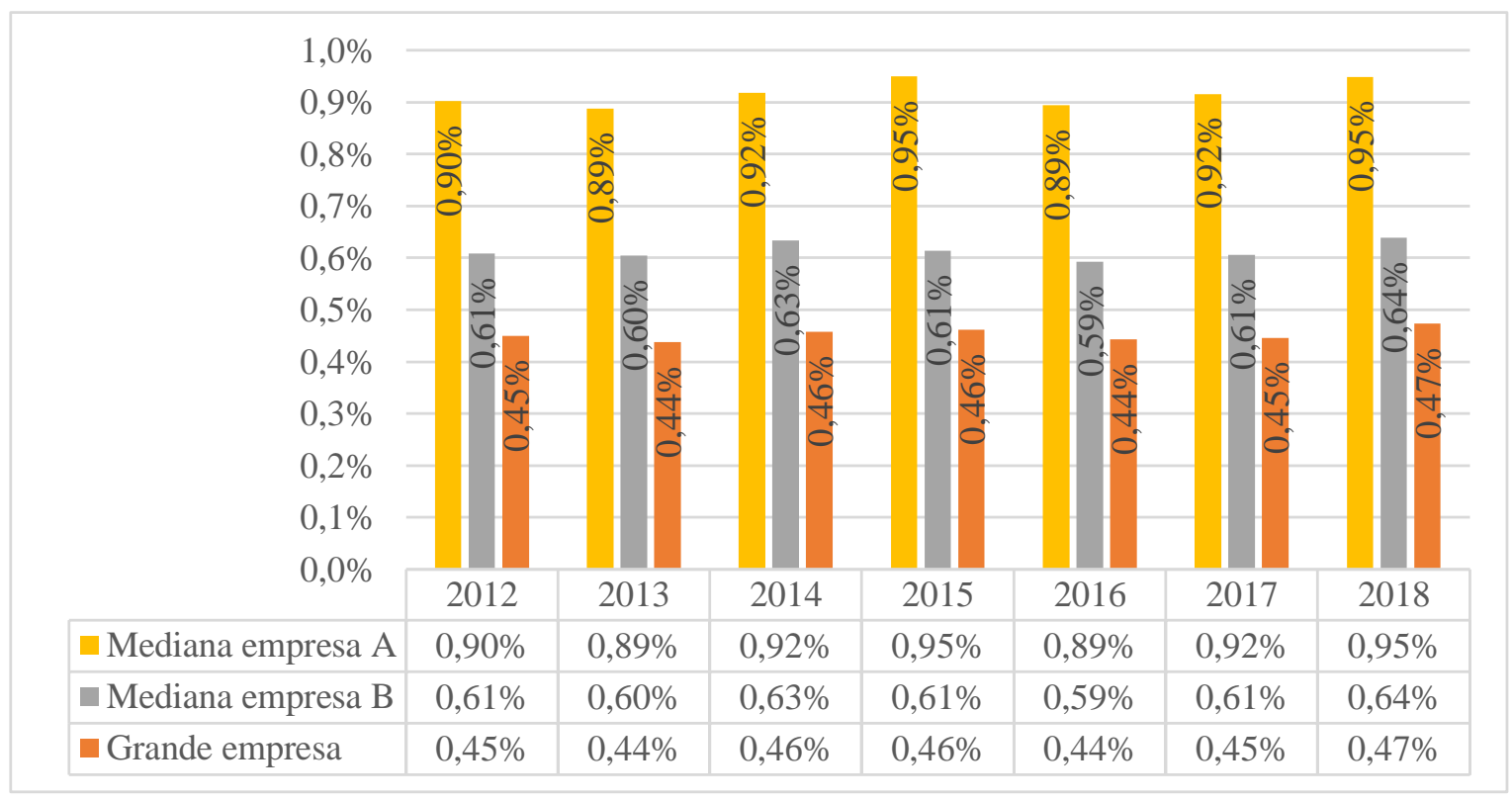

Fuente: Elaboración propia con datos del Instituto Nacional de Estadística y Censos (2019)

En Ecuador, las medianas y grandes empresas, conforman aproximadamente el 2\% del sector empresarial y aun así son fuentes generadoras de empleos, la figura 1 indica una tendencia constante durante el periodo 2012-2018, dado a las cambiantes reformas tributarias y pago de impuestos; según Mayorga et al. (2020) la eliminación del anticipo de impuesto a la renta es una fortaleza para las empresas, ya que esto les genera pérdida de liquidez, así mismo aquellas empresas que hayan generado pérdidas también estaban obligadas al pago del impuesto, independientemente de su situación financiera. En el sector empresarial nacional, la mediana empresa A representa aproximadamente el $0,90 \%$, la mediana empresa B mantiene una equivalencia alrededor del $0,60 \%$ y las grandes empresas corresponden en promedio al $0,45 \%$. 


\section{Tabla 1}

Principales características de estudios que analizan la contingencia estructural

\begin{tabular}{lcccc}
\hline \multicolumn{1}{c}{ Autores } & $\begin{array}{c}\text { Periodo de } \\
\text { estudio }\end{array}$ & $\begin{array}{c}\text { Método de } \\
\text { estimación }\end{array}$ & $\mathbf{R}^{\mathbf{2}}$ & $\begin{array}{c}\text { Pruebas } \\
\text { estadísticas }\end{array}$ \\
\hline $\begin{array}{l}\text { Blanco, Vásquez, García, y } \\
\text { Melamed (2020) }\end{array}$ & 2019 & $\begin{array}{c}\text { Análisis de } \\
\text { varianza }\end{array}$ & 0,989 & Alfa de Cronbach \\
\hline $\begin{array}{l}\text { López, Rodríguez y Trujillo } \\
(2021)\end{array}$ & 2016 & $\begin{array}{c}\text { Regresión } \\
\text { múltiple }\end{array}$ & 0.557 & $\begin{array}{c}\text { Correlación de } \\
\text { Pearson }\end{array}$ \\
\hline $\begin{array}{l}\text { Vicuña, Cortez y Basurto } \\
\text { (2018) }\end{array}$ & $2017-2018$ & $\begin{array}{c}\text { Regresión } \\
\text { múltiple }\end{array}$ & 0,948 & $\begin{array}{c}\text { Sétodo Best } \\
\text { Subset Selection }\end{array}$ \\
\hline
\end{tabular}

Fuente: Elaboración propia

En la tabla 1 se expresan las principales características de la incidencia de la contingencia estructural en la competitividad empresarial. Un estudio realizado por Blanco et al. (2020) en el sector de alimentos de la ciudad de Barranquilla; toman como variables de estudio a la estructura organizativa y la competitividad en un modelo econométrico ANOVA, donde logran determinar que un mayor nivel de estructuración, permite un mayor nivel de competitividad en la organización. Según López, Rodríguez y Trujillo (2021) indican que la gestión empresarial se desenlaza en la correcta gestión estratégica, operativa y financiera de la empresa, y explican mediante un modelo de regresión lineal que la gestión empresarial influye en $50.6 \%$ en la competitividad.

En referencia al inciso de Vicuña, Cortez y Basurto (2018) mencionan que la competitividad depende de la administración interna específicamente del nivel y uso del equipo informático, ventas por trabajadores, materia prima utilizada, mano de obra y gastos varios; los autores compilaron un modelo de regresión lineal para corroborar lo antes mencionado, según las pruebas estadísticas el $\mathrm{R}^{2}$ es cercano a uno, mantiene comportamiento normal en la distribución de los residuos y las variables son significativas. En definitiva, según los 3 incisos anteriores, la administración empresarial incide en el desempeño competitivo, de esta manera la estructura organizativa interviene en el desarrollo y capacidad de competencia empresarial, además mantiene la participación de las empresas mediante la eficiencia de las áreas funcionales en la industria permitiendo el desarrollo empresarial.

\section{Materiales y Métodos}

Enfoque de investigación cuantitativo de alcance correlacional con diseño transversal (Bernal C. , 2010). Este proceso permitió analizar sistemáticamente un determinado grupo socioeconómico en un tiempo único, que mediante el uso de la estadística se midió con precisión 
Ingrid Batallas Astudillo, Arelys Luna y Patricia Uriguen Aguirre.

ISSN 2477-9024. Innova Research Journal (Mayo-Agosto, 2021). Vol 6, No. 2, pp. 209-224

la causalidad e incidencia de la variable de interés con las variables independientes (Hernández et al., 2010). En primera instancia, se manipuló la base de datos de ENESEM del INEC del periodo 2018, se segmentó a la provincia de El Oro mediante el programa estadístico Stata, se obtuvo como resultado 120 observaciones, se elaboraron diferentes modelos econométricos de regresión múltiple lineal que cumplan con los supuestos de validación, con la finalidad de estimar la incidencia de la contingencia estructural en el nivel de ingresos de las medianas y grandes empresas orenses.

Según Wooldridge (2010) la validación de un modelo de regresión lineal múltiple requiere aprobar los supuestos de normalidad de residuos, homocedasticidad de los residuos y colinealidad. La aplicación de los supuestos permitió identificar que los residuos promediaron por debajo y encima de la media; en el mismo sentido, la varianza se situó a la misma o similar distancia. Los apartados de Vilá et al. (2019) mencionaron que el supuesto de colinealidad perturbe a la estimación, debido a la correlación entre 2 o más variables. Por ende, las variables independientes, en su medida, estaban parcialmente correlacionadas entre sí. Para elegir el modelo econométrico con mejor ajuste se utiliza el criterio de información Bayesiano (BIC) y el criterio de información Akaike (AIC). El modelo aceptado es el que refleje menor valor en los criterios (Hernández et al., 2019).

\section{Resultados}

De acuerdo a lo recabado en la data, se manifestó como principales resultados que, la estructura organizacional como enfoque contingente predomina en el desempeño de las medianas y grandes empresas de la provincia de El Oro. Para la elección del modelo de regresión lineal múltiple se aplicaron diferentes pruebas y métodos estadísticas tales como análisis del $\mathrm{R}^{2}$, prob $>\mathrm{F}$, p-valúe y los test de normalidad, homocedasticidad y colinealidad para identificar que variables de contingencia estructural explican el nivel de ingresos de las medianas y grandes empresas orenses. Gujarati (2010) menciona que la variable dependiente o regresada en los modelos de regresión depende de dos o más variables independientes o regresoras. A priori, se seleccionaron las variables que estiman el nivel de ingreso empresarial orense.

\section{Tabla 2}

Modelos de regresión múltiple

\begin{tabular}{cccc}
\hline Modelos múltiples & F & Prob>F & $\mathbf{R}^{2}$ \\
\hline Modelo 1 & 2121.25 & 0.0000 & 0.9956 \\
Modelo 2 & 7789.53 & 0.0000 & 0.9994 \\
Modelo 3 & 8602.78 & 0.0000 & 0.9994 \\
\hline \multicolumn{4}{l}{ Fuente: Elaboración propia con datos de ENESEM }
\end{tabular}

Se determinaron 3 modelos de regresión múltiple, de acuerdo con el coeficiente de correlación $\mathrm{R}^{2}$, los tres modelos tienen un valor cercano a 1 , es decir existe un correcto y específico ajuste lineal en los parámetros, sin embargo, puede existir un problema de colinealidad. La probabilidad $\mathrm{F}$ es menor a 0.05 , lo cual indica que las variables en su conjunto explican cada 
modelo. El valor F corrobora la prob>F, entre más alto sea su valor las variables seleccionadas son apropiadas. Es probable que el segundo y tercer modelo sean los adecuados para el estudio, dado que la tabla 2 indica que los modelos 2 y 3 tienen una distribución F de 7789.53 y 8602.78 respectivamente.

Aplicando los supuestos para que los modelos sean validados en la normalidad y homocedasticidad en los residuos su p-valúe debe ser mayor a 0.05. El índice de factor de inflación de la varianza (VIF) debe ser menor a 10 para que la colinealidad no sea preocupante, no exista una proximidad correlacional entre las variables de estudio (Herrera et al., 2020).

El modelo 1 refleja una normalidad con un p-valúe de 0.00, tiene una homocedasticidad de 0.9882, además el VIF muestra un valor de 2.65. Por otra parte, el modelo 2 arroja una normalidad de 0.4009 , con colinealidad de 2.80, empero, la homocedasticidad no posee una matriz positiva, es decir la correlación no cumple con las condiciones o es posible que existan variables con observaciones faltantes o con valores negativos. Por último, el modelo 3 tiene 0.3530 de normalidad, 0.3093 en homocedasticidad y 2.54 en VIF. Por tanto, los supuestos de normalidad y homocedasticidad en residuos y colinealidad validan el modelo 3, tal como indica la tabla 3, existe una distribución normal y varianza constante en las observaciones, en otros términos, los datos guardan similitud en la distancia.

\section{Tabla 3}

Supuestos de modelo de regresión múltiple

\begin{tabular}{cccc}
\hline $\begin{array}{c}\text { Modelos } \\
\text { múltiples }\end{array}$ & $\begin{array}{c}\text { Normalidad en } \\
\text { residuos }\end{array}$ & $\begin{array}{c}\text { Homocedasticidad de } \\
\text { residuos }\end{array}$ & Colinealidad \\
\hline Modelo 1 & 0.0000 & 0.9882 & 2.65 \\
Modelo 2 & 0.4009 & matrix not positive definite & 2.80 \\
Modelo 3 & 0.3530 & 0.3093 & 2.54 \\
\hline
\end{tabular}

Fuente: Elaboración propia con datos de ENESEM

Además de comparar y elegir el modelo consistente y el que mejor se ajusta, se corrobora el inciso anterior mediante la aplicación de los criterios de información BIC y AIC, la tabla 4 muestra que los valores más bajos se sitúan en el modelo 3, efectivamente, es el que mejor se ajusta para determinar que variables vinculadas con la contingencia estructural explican el nivel de ingresos de las medianas y grandes empresas de la provincia de El Oro.

\section{Tabla 4}

Criterios de información BIC y AIC

\begin{tabular}{cccc}
\hline Modelos múltiples & Modelo 1 & Modelo 2 & Modelo 3 \\
\hline BIC & 2989.8555 & 1978.5095 & 1974.7419 \\
AIC & 2964.3167 & 1950.044 & 1948.4661 \\
\hline
\end{tabular}


Ingrid Batallas Astudillo, Arelys Luna y Patricia Uriguen Aguirre.

ISSN 2477-9024. Innova Research Journal (Mayo-Agosto, 2021). Vol 6, No. 2, pp. 209-224

Fuente: Elaboración propia con datos de ENESEM

La tabla 5 indica la abreviatura y significado de las variables independientes que explican el nivel de ingresos de las medianas y grandes empresas orenses que se utilizaron en el modelo de regresión lineal múltiple.

\section{Tabla 5}

\section{Variables de estudio}

\begin{tabular}{ll}
\hline Abreviatura & \multicolumn{1}{c}{ Significado de variables } \\
\hline v1001 & Total de ingresos \\
v5013 & Total de directores y gerentes \\
v5065 & Total de técnicos actividades administrativas, financieras y apoyo logístico \\
v5104 & Total de técnicos en equipo informático, y en procesamiento de base de datos \\
v5130 & Total de técnicos en soporte de redes y soporte computacional \\
repacces & Suministros, herramientas, materiales y repuestos \\
vtagua & Valor total de agua \\
vtee & Valor total de energía eléctrica \\
totinsum & Total insumos \\
v9111 & Valor total combustibles y lubricantes \\
v1042 & Total costo y gasto \\
v11053 & Educación Superior - Carencia de habilidades de trabajo o rasgos personales \\
\hline
\end{tabular}

Fuente: Elaboración propia con datos de ENESEM

Las variables del modelo econométrico con un p-valúe menor al 0.05 tienen mayor incidencia y significancia estadística sobre el nivel de ingreso de las medianas y grandes empresas en la provincia de El Oro, tal como se indica en la tabla 6. El total de directores y gerentes, valor total en agua, costos y gastos tienen un p-valúe de 0.000. Asimismo, las variables explicativas: técnicos en soporte de redes y soporte computacional, técnicos en equipo informático y en procesamiento en base de datos, y valor total en combustibles y lubricantes tienen un p-valúe de $0.002,0.002$ y 0.004 correspondientemente.

El total de técnicos en actividades administrativas, financieras y apoyo logístico, el valor total de energía eléctrica, la carencia de habilidades de trabajo o rasgos personales, y los suministros, herramientas, materiales y repuestos se sitúan con un p-valúe de 0.014, 0.023, 0.028 y 0.034 respectivamente. Sin embargo, el total de insumos no guardan significancia estadística, dado que su p-valúe es 0.659 , valor que supera el 0.05 , pero ayuda en su conjunto a explicar el modelo econométrico.

Con respecto a los recursos naturales utilizados por las empresas, se determina que por cada dólar invertido en combustible y lubricantes para fines productivos los ingresos de las medianas y grandes empresas incrementan en $\$ 2,62$ y por cada dólar invertido en el uso del agua los ingresos de las empresas aumentan en \$5,54. Sin embargo, por cada dólar ocupado en energía eléctrica los ingresos de las empresas disminuyen $\$ 1,17$. La variable insumos tiene una 
probabilidad mayor al $5 \%$ y genera un pequeño aumento en los ingresos de las empresas de $\$ 0,01$, por tanto la variable no es económica ni estadísticamente significativa.

\section{Tabla 6}

Análisis de regresión múltiple

\begin{tabular}{rrrrrrr}
\hline Total_Ingresos & \multicolumn{1}{c}{ Coef. } & Std. Err. & \multicolumn{1}{c}{$\mathbf{t}$} & \multicolumn{1}{c}{$\mathbf{P}>|\mathbf{z}|$} & \multicolumn{2}{c}{ [95\% Conf. Interval] } \\
\hline v5013 & 79761.72 & 19454.45 & 4.10 & 0.000 & 40757.90 & 118765.54 \\
v5065 & 44404.65 & 17504.86 & -2.54 & 0.014 & -79499.77 & -9309.52 \\
v5104 & 538121.92 & 167810.58 & 3.21 & 0.002 & 201681.96 & 874561.89 \\
v5130 & -820702.70 & 253504.96 & -3.24 & 0.002 & -1328950 & -312455.9 \\
repacces & -0.10 & 0.05 & -2.18 & 0.034 & -0.20 & -0.01 \\
vtagua & 5.54 & 0.86 & 6.47 & 0.000 & 3.82 & 7.25 \\
vtee & -1.17 & 0.50 & -2.33 & 0.023 & -2.17 & -0.16 \\
totinsum & 0.01 & 0.01 & 0.44 & 0.659 & -0.02 & 0.03 \\
v9111 & 2.62 & 0.87 & 3.02 & 0.004 & 0.88 & 4.36 \\
v1042 & 1.01 & 0.01 & 127.40 & 0.000 & 1.00 & 1.03 \\
v11053 & 383438.51 & 169992.60 & 2.26 & 0.028 & 42623.88 & 724253.15 \\
const & -453578.2 & 244772.7 & -1.85 & 0.069 & -944317.9 & 37161.59
\end{tabular}

Fuente: Elaboración propia con datos de ENESEM

Por cada dólar financiado en los suministros, herramientas, materiales y repuestos, la variable reduce ligeramente los ingresos anuales a $\$ 0.10$. Los costos y gastos contribuyen al incremento de los ingresos de las empresas en \$1.01 por cada dólar desembolsado. Bajo otro punto de vista, a pesar que los trabajadores jóvenes con título de tercer nivel presenten carencia de rasgos personales, estos contribuyen a la empresa, a que los ingresos aumenten en $\$ 383.438,51$.

Por cada director o gerente que se incorpore en las empresas aumentan los ingresos totales anuales en \$79.761,72. Asimismo, por cada personal técnico en equipo informático y en procesamiento en base de datos; y técnicos administrativos, financieros y logísticos contratados por la empresa, los ingresos aumentan $\$ 538.121,92$ y $\$ 44.404,65$ respectivamente. Por último, por cada profesional técnico en soporte de redes y computacional que se incorpore en la empresa contrae los ingresos en $\$ 820.702,70$.

\section{Conclusiones}

La teoría de contingencia es un factor que incide en los ingresos de las empresas de la provincia de El Oro, debido a que los resultados obtenidos evidencian el cumplimiento del objetivo de la investigación. El modelo de regresión múltiple que cumple los supuestos de normalidad, homocedasticidad y colinealidad, con mejor bondad de ajuste, distribución $\mathrm{F}$ y significancia estadística determinó que las variables que inciden en los ingresos de las medianas y grandes empresas de la provincia de El Oro se centran en el personal directivo y gerencial; técnicos en equipo informático y en procesamiento en base de datos, técnicos administrativos, financieros y logísticos, en los costos y gastos totales e importes en el consumo de agua, combustible y lubricantes. 
Ingrid Batallas Astudillo, Arelys Luna y Patricia Uriguen Aguirre.

ISSN 2477-9024. Innova Research Journal (Mayo-Agosto, 2021). Vol 6, No. 2, pp. 209-224

Dado a la actividad comercial y agrícola en la provincia, las medianas y grandes empresas deben enfocarse en invertir en el uso eficiente del agua, combustible y lubricantes, debido a que cuando producen, transportan y comercializan más productos generan mayores ventas y por ende ingresos a las empresas. Asimismo, las empresas orenses deben orientarse a reclutar personal especializado en funciones gerenciales, administrativas e informáticas. En sí, la empresa no debe enfocarse en la carencia de habilidades de rasgo personal, es decir como el personal novicio se relaciona y actúa en el contexto social, pues estos trabajadores aportan al incremento de los ingresos económicos. Ciertamente, las empresas deben contratar personal que no carezca de habilidades en el desempeño laboral para que no ocasione pérdidas económicas.

Se evidencia que la gestión del capital humano se enfatiza en el personal profesional especializado en las áreas de gerencia, administración, financiera, logística e informática; el capital humano tiene una contribución significativa en los ingresos económicos de las empresas orenses, dado a los conocimientos, capacidades de liderazgo, toma de decisiones, incorporación de IDi para generar ventaja competitiva y posicionamiento en el mercado. Las estrategias gerenciales a través del uso eficiente y óptimo de los bienes de capital y de los recursos materiales y naturales generan también un aporte en los ingresos de las empresas mediante la optimización en las actividades productivas y control de costos y gastos de las empresas.

En efecto, de acuerdo al análisis de contingencia las medianas y grandes empresas de la provincia de El Oro deben implementar estrategias de estructura organizativa que permitan crear valor y maximizar los beneficios y ganancias de las empresas. Las medianas y grandes empresas orenses forman parte del crecimiento económico de la provincia de El Oro, el enfoque contingente demuestra que no existe una estructura organizativa ideal para las empresas, esto debido a factores como el tipo de actividad que lleve a efecto la empresa, además de su tamaño y el entorno ambiental en el que se desarrolla. En definitiva, el aporte de la teoría de contingencia dentro de las organizaciones permite identificar la forma eficiente en la que se debe manejar los recursos y la toma de decisiones.

\section{Referencias bibliográficas}

Apolo, N., Uriguen, P., \& Ochoa, V. (2018). Evolución del sector empresarial de la provincia de El Oro. En L. Capa, J. Sotomayor, y F. Vega (Eds.), La Provincia de El Oro algunas consideraciones de los sectores productivos y empresariales (págs. 31-53). Editorial UTMACH.

Bernal, C. (2010). Metodología de la investigación. PEARSON EDUCACIÓN.

Blanco, A., Vásquez, Á., García, R., \& Melamed, E. (2020). Estructura organizacional como determinante competitivo en pequeñas y medianas empresas del sector alimentos. Revista de Ciencias Sociales (RCS), 26(42), 133-147. https://doi.org/10.31876/rcs.v26i2.32429 
Bravo-Macías, C., Márquez-Bravo, Y., \& Saltos-Solórzano, J. (2016). El comportamiento organizacional y los procesos productivos en las MIPYMES. Dominio de las Ciencias, 2(4), 408-420. http://dx.doi.org/10.23857/dc.v2i4.263

Carvallo, Y., Gonzaga, S., Urdaneta, A., \& García, M. B. (2020). Competitividad en las MIPYMES. Propuestas de mejoras en los procesos administrativos y organizacionales de la Provincia de El Oro- Ecuador. Revista de Humanidades y Ciencias Sociales y Multidisciplinaria, 7(1), 18-34. http://100cs.cl/index.php/cs/article/view/191

Cedeño, A., Asencio, L., \& Villegas, M. (2019). Las estrategias gerenciales como base fundamental para la administración en los negocios. Universidad y Sociedad, 11(5), 191200. https://rus.ucf.edu.cu/index.php/rus/article/view/1360

Código Orgánico de la Producción, Comercio e Inversiones. (2018). Reglamento de Inversiones del Código Orgánico de la Producción (Ultima modificación: 20-dic.-2018). Asamblea Nacional del Ecuador. https://www.produccion.gob.ec/wpcontent/uploads/2019/05/Literal-3.-Reglamento-del-Codigo-Orgaanico-de-laProduccioon-Comercio-e-Inversiones-COPCI.pdf

De la Torre, M. (2018). Gestión del riesgo organizacional de fraude y el rol de Auditoría Interna. $\begin{array}{llll}\text { Revista Contabilidad } \quad y \quad \text { Negocios, } & \text { 13(25), }\end{array}$ https://doi.org/10.18800/contabilidad.201801.004

Delfín, F., \& Acosta, M. (2016). Importancia y análisis del desarrollo empresarial. Revista Pensamiento y Gestión (40), 184-202. http://dx.doi.org/10.14482/pege.40.8810

Dias, G., Camargo, T., \& Zanin, A. (2017). Competitividad del mercado y gestión de beneficios: un estudio desde la teoría de la contingencia. Revista de Ciencias de la Administración, 19(49), 86-101. https://doi.org/10.5007/2175-8077.2017v19n49p86

Duran, S., Crissien, J., Virviescas, J., \& García, J. (2017). Estrategias gerenciales para la formación de equipos de trabajos en empresas constructoras del Caribe colombiano. Revista ESPACIOS, 38(13), 24-39. https://www.revistaespacios.com/a17v38n13/a17v38n13p24.pdf

Facin, C., Gorla, M., Lavarda, R., \& Angonese, R. (2016). The Influence of Contingency Factors on the Development of a Budgeting System in a Brazilian Textile Manufacturing Company. Revista Gestão Organizacional, 19(1), 115-124. http://dx.doi.org/10.21714/1679-18272016v14n1.p115-124

Galarza, S., García, J., Ballesteros, L., Cuenca, V., \& Fernández, A. (2017). Estructura organizacional y estilos de liderazgo en Cooperativas de Ahorro y Crédito de Pichincha. Revista de Cooperativismo y Desarrollo, 5(1), 19-31. http://coodes.upr.edu.cu/index.php/coodes/article/view/156

Gonzáles, W. (2016). La implementación de procesos de informatización en organizaciones como competencia en la formación de profesionales en informática. Revista e-Ciencias de la Información, 6(2), 1-18. http://dx.doi.org/10.15517/eci.v6i2.25276

Graneroa, L., Guillénb, M., \& Bañón, A. (2017). Influencia de los factores de contingencia en el desarrollo del cuadro de mando integral y su asociación con un rendimiento mejor. El caso de las empresas españolas. Revista de Contabilidad, 20(1), 82-94. http://dx.doi.org/10.1016/j.rcsar.2016.07.002 
Ingrid Batallas Astudillo, Arelys Luna y Patricia Uriguen Aguirre.

ISSN 2477-9024. Innova Research Journal (Mayo-Agosto, 2021). Vol 6, No. 2, pp. 209-224

Gujarati, D. (2010). Econometría. McGraw-Hill / Interamericana Editores.

Hernández, J., Tamarit, J., García, X., Hernández, A., Reynoso, R., \& Reyes, V. (2019). Modelos alométricos altura-diámetro para Bucida buceras (pukté) en Quintana Roo, México. Revista BOSQUE, 40(3), 267-276. https://dx.doi.org/10.4067/S0717-92002019000300267

Hernández, O. (2016). La auditoría interna y su alcance ético empresarial. Revista Actualidad Contable FACES, 19(33), 15-41. http://www.saber.ula.ve/handle/123456789/42077

Hernández, R., Fernández, C., \& Baptista, M. d. (2010). Metodología de la investigación. McGraw-Hill / Interamericana Editores.

Herrera, M., Gutiérrez, M., Moreno, A., \& Segura, J. (2020). Factores de capital humano que impactan el capital social: estudio de hoteles del sur de Tamaulipas. Revista CienciaUAT, 15(1), 147-161. https://doi.org/10.29059/cienciauat.v15i1.1402

Hinojosa, C., Limón, E., \& Navarrete, C. (2020). Cultura empresarial en las pymes del municipio de Tamazula de Gordiano, Jalisco (México). Revista Ánfora, 27(49), 143-172. https://doi.org/10.30854/anf.v27.n49.2020.743

INEC. (10 de Diciembre de 2019). Cerca de 900 mil empresas se registraron en Ecuador en 2018. Instituto Nacional de Estadística y Censo: https://www.ecuadorencifras.gob.ec/cerca-de900-mil-empresas-se-registraron-en-ecuador-en-2018/

Instituto Nacional de Estadística y Censo. (2019). Directorio de Empresas y Establecimientos (Boletín técnico $N^{\circ}$ 01-2020-DIEE). Dirección de Estadísticas Económicas (DECON). Gestión de Estadísticas de Empresas en base a Registros Administrativos. Estadísticas de las empresas: https://www.ecuadorencifras.gob.ec/documentos/webinec/Estadisticas_Economicas/DirectorioEmpresas/Directorio_Empresas_2019/Boletin_T ecnico_DIEE_2019.pdf

King, K., \& Vaiman, V. (2019). Enabling effective talent management through a macro-contingent approach: A framework for research and practice. Business Research Quarterly Journal, 22(3), 194-206. https://doi.org/10.1016/j.brq.2019.04.005

López, R., Rodríguez, L., \& Trujillo, G. (2021). La gestión empresarial y su influencia en la competitividad de las microempresas del sector pesquero de la ciudad de Piura. Ciencia $\begin{array}{lllll}\text { Latina Revista } \quad \text { Científica } & \text { Multidisciplinar, } & 5(1), & 485 .\end{array}$ https://doi.org/10.37811/cl_rcm.v5i1.245

Luna, C., De la Hoz, R., Gómez, A., Manjarrés, A., Vidal, J., Jaramillo, O., \& Berdugo, C. (2016). Universidad corporativa y aprendizaje organizacional: un marco de referencia. Revista Dirección y Organización, 58, 79-94. https://revistadyo.es/index.php/dyo/article/view/488

Mayorga, T., Campos, L., Arguello, C., \& Villacis, J. (2020). Nuevas reformas tributarias y su impacto en la economía del Ecuador. Revista Científica FIPCAEC, 5(18), 30-40. https://fipcaec.com/index.php/fipcaec/article/view/199

Méndez, S., Morua, J., \& Hernández, R. (2018). Complejidad y dinámica, la necesidad de considerarlas en la evaluación organizacional. Revista de Ciencias Sociales (RCS), 24(2), 9-23. https://produccioncientificaluz.org/index.php/rcs/article/view/24816

Esta obra se comparte bajo la licencia Creative Common Atribución-No Comercial 4.0 International (CC BY-NC 4.0) Revista de la Universidad Internacional del Ecuador. URL: https://www.uide.edu.ec/ 
Montoya, C., \& Boyero, M. (2016). El recurso humano como elemento fundamental para la gestión de calidad y la competitividad organizacional. Revista Científica "Visión de Futuro", 20(2), 1-20. https://visiondefuturo.fce.unam.edu.ar/index.php/visiondefuturo/article/view/65

Oña, B. (2020). Teoría de la Contingencia y Teoría de Costos en sectores económicos vulnerables. Revista Arbitrada Interdisciplinaria Koinonía, 5(4), 706-716. https://fundacionkoinonia.com.ve/ojs/index.php/revistakoinonia/article/view/1004

Palacio, A., \& Núñez, M. (2020). Administración del riesgo estratégico en grandes empresas privadas de Colombia. AD-minister (36), 67-96. https://doi.org/10.17230/Ad-minister.36.4

Pérez, L., Guillén, M., \& Bañón, A. (2017). Influencia de los factores de contingencia en el desarrollo del cuadro de mando integral y su asociación con un rendimiento mejor. El caso de las empresas españolas. Revista de Contabilidad, 20(1), 82-94. http://dx.doi.org/10.1016/j.rcsar.2016.07.002

Ponce, G., Espinoza, D., Ríos, J., \& Tapia, K. (2017). Capacidades organizacionales generadoras de valor: análisis del sector industrial. RETOS Revista de Ciencias de la Administración y Economía, 7(13), 143-162. https://doi.org/10.17163/ret.n13.2017.09

Ramírez, A., Ramírez, R., \& Calderón, E. (2017). La gestión administrativa en el desarrollo empresarial. Revista Contribuciones a la Economía, 1-20. http://eumed.net/ce/2017/1/gestion.html

Ramírez, R., Chacón, H., \& Valencia, K. (2018). Gestión del talento humano como estrategia organizacional en las pequeñas y medianas empresas. Revista del Centro de Investigación de Ciencias Administrativas y Gerenciales, 16(1), 20-42. http://ojs.urbe.edu/index.php/cicag/article/view/2929

Segredo, A. (2016). Aproximación teórica a la evolución, teorías, enfoques y características que han sustentado el desarrollo de las organizaciones. Revista Cubana de Salud Pública, 42(4), 585-595. http://scielo.sld.cu/scielo.php?script=sci_arttext\&pid=S086434662016000400009\&lng=es\&tlng=es

Serrano, P., Señalin, L., Vega, F., \& Herrera, J. (2018). El control interno como herramienta. Revista ESPACIOS, 39(03),

30 . https://www.revistaespacios.com/a18v39n03/a18v39n03p30.pdf

Sotomayor, J., Apolo, N., \& Quispe, J. (2018). Estructura Social y Económica de la Provincia de El Oro. En L. Capa, J. Sotomayor, y F. Vega (Eds.), La Provincia de El Oro algunas consideraciones de los sectores productivos y empresariales (págs. 15-29). Editorial UTMACH.

Uriguen, P., Morán, G., \& Ochoa, G. (2018). Estructura Administrativa del Sector Empresarial de la provincia de El Oro. En L. Capa, J. Sotomayor, y F. Vega (Eds.), La Provincia de El Oro algunas consideraciones de los sectores productivos y empresariales (págs. 55-75). Editorial UTMACH.

Valle, A., Proaño, T., \& Cruz, M. (2017). Estructura, Cultura y Cambio organizacional Cultura Cambio - Forma - Fuerza. Revista Científica Hermes, 28, 304-324. https://doi.org/10.21710/rch.v18i0.343

Vicuña, A., Cortez, B., \& Basurto, Y. (2018). Determinación de modelos predictivos para los indicadores de competitividad empresarial aplicando regresión lineal. Iberian Journal of 
Ingrid Batallas Astudillo, Arelys Luna y Patricia Uriguen Aguirre.

ISSN 2477-9024. Innova Research Journal (Mayo-Agosto, 2021). Vol 6, No. 2, pp. 209-224

Information Systems and Technologies(E18), 94-107. https://search.proquest.com/openview/4a79f8c408fbf627976d3b25b1cc0bef/1?pqorigsite $=$ gscholar $\&$ cbl $=1006393$

Vilá, R., Torrado, M., \& Reguant, M. (2019). Análisis de regresión lineal múltiple con SPSS: un ejemplo práctico. Revista de Innovación e Investigación en Educación, 12(2), 1-10. http://doi.org/10.1344/reire2019.12.222704

Vivar, A., Erazo, J., \& Narváez, C. (2020). La cadena de valor como herramienta generadora de ventajas competitivas para la Industria Acuícola. Revista Arbitrada Interdisciplinaria KOINONIA, 5(10), 4-33. http://dx.doi.org/10.35381/r.k.v6i10.686

Wooldridge, J. (2010). Introducción a la econometría: Un enfoque moderno. Cengage Learning Editores.

Zapata, G. (2015). El tamaño y la estructura de la organización: un estudio teórico y empírico con el enfoque contingente. Revista Ciencias Estratégicas, 23(34), 193-208. https://www.redalyc.org/articulo.oa?id=1513/151350864003

Zapata, G., Sigala, L., \& Canet, M. (2016). Características de diseño organizativo de las medianas empresas del estado Lara, Venezuela. Revista Venezolana de Gerencia, 21(74), 288-308. https://www.redalyc.org/pdf/290/29046685008.pdf 